\title{
Synthesis of Pullulan-Mediated Silver Nanoparticles (AgNPs) and their Antimicrobial Activities
}

\author{
Yusra Al-Owasi ${ }^{1}$, Abdulkhadir Elshafie ${ }^{2}$, Nallusamy Sivakumar ${ }^{2 *}$ and Saif N. Al-Bahry ${ }^{2}$
}

${ }^{1}$ Department of Biochemistry, Sultan Qaboos University Hospital, Sultan Qaboos University, P.O Box 36, PC 123, Al- Khoud, Muscat; ${ }^{2}$ Department of Biology, College of Science, Sultan Qaboos University, P.O Box 36, PC 123, Al- Khoud, Muscat. *Email: apnsiva@squ.edu.om.

\begin{abstract}
The synthesis of silver nanoparticles (AgNPs), using plant extracts, bacteria, fungi and yeasts, and their antimicrobial activities have been widely investigated and well documented. However, pullulan AgNPs and their antimicrobial activities have not received much attention. The objective of this study was to synthesize pullulan AgNPs, characterize them, and test their antibacterial and antifungal activities. Pullulan was extracted from Aureobasidium mangrovei isolated from Oman and, using UV-Vis spectroscopy and Fourier Transform Infrared (FTIR), found to be identical to the commercial pullulan obtained from Sigma, USA. Transmission electron microscopy (TEM) showed that most of the synthesized particles were poly-dispersed, irregular in shape, and most were spherical with an average size of $9.76 \mathrm{~nm}$. Pullulan-mediated AgNPs were found to have antibacterial activities, and the ANOVA test showed that there were no significant differences between $\mathrm{AgNO}_{3}$, Pullulan and pullulan-mediated AgNPs for all the bacteria tested. Pullulan-mediated nanoparticles were found to have antifungal activity against Curvularia lunata, Fusarium incarnatum, Aspergillus niger, Aspergillus flavus, Aspergillus ochraceus and Penicillium sp. The ANOVA test also revealed that there was a significant difference in antifungal activity between pullulan and pullulan-mediated AgNPs, pullulan-mediated nanoparticles having shown a higher inhibitory activity than pullulan. Pullulan and pullulan-mediated nanoparticles could be used in the food industry and are safer than silver nitrates.
\end{abstract}

Keywords: Pullulan; Pullulan-mediated silver nanoparticles; Antibacterial and antifungal activities; Aureobasidium mangrovei.

$$
\text { إنتاج الجسيمات النانويه الفضيه من البوليولان و أنشطتها المضاده للمكيروبات }
$$

$$
\text { يسرى العويسي، عبدالقادر الشفيع، سيفا كومار و سيف البحري }
$$

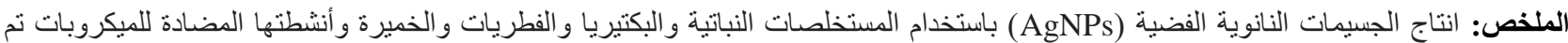

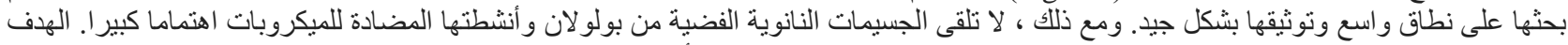

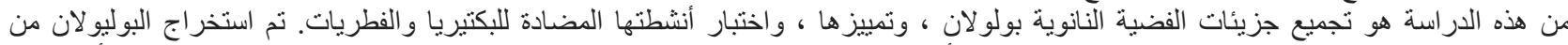

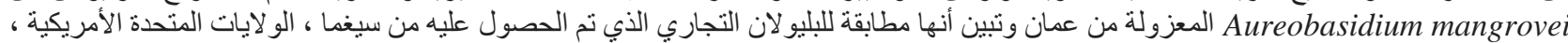

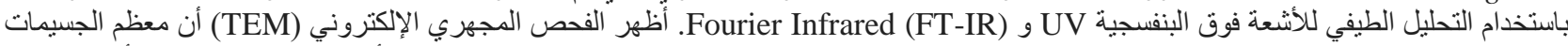

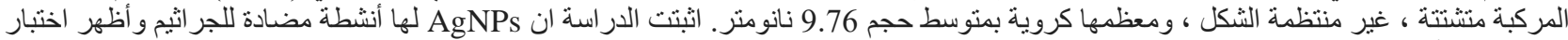

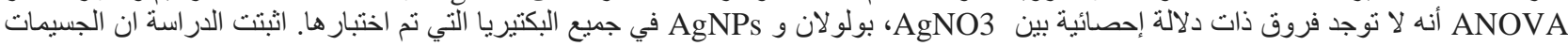

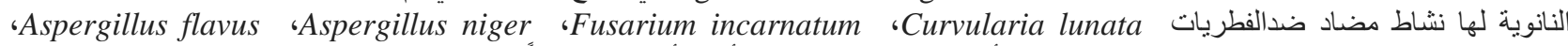
Penicillium sp و Aspergillus ochraceus AgNPs

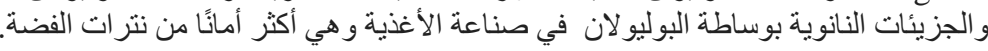
الكلمات المفتاحية: الجسيمات النانوية الفضية، بولو لان، انتاج، أنشطة مضادة للميكروبات. 


\section{SYNTHESIS OF PULLULAN MEDIATED-SILVER NANOPARTICLES (AGNPS)}

\section{Introduction}

$\mathbf{T}$

The antibacterial and antifungal properties of AgNPs have been used for many purposes, such as in biomedical and scientific applications, water purification, and food preservation [1]. This is because of their low toxicity to animal cells and high toxicity to microorganisms. Nanoparticles have a broad spectrum of antibacterial and antifungal activities and have not been associated yet with microbial resistance [2]. They have the potential of being used against multi-drug resistant microbial strains.

The synthesis of AgNPs using plant extracts, bacteria, fungi, yeasts and their antimicrobial activities has been widely investigated [3,4] and there is a growing interest in the synthesis of nanoparticles from polymers such as polyester, cellulose, starch, dextran, alginate, and agar [2,6]. The combined efficacy of synthesized Aspergillus flavus nanoparticles and different antibiotics against multi-drug resistant bacteria has been reported [5]. However, there is still very little information available on pullulan-mediated nanoparticles and their antimicrobial activities. The synthesized and characterized pullulan-mediated nanoparticles and their antibacterial activities against Escherichia coli, Pseudomonas aeruginosa, Listeria monocytogenes and against fungal isolates Aspergillus spp. and Penicillium spp. have been reported [6] and in addition there has been some study [2] on the activity of transparent nanocomposite films of pullulan and AgNPs against Aspergillus niger, which has shown that the film can be used as an antifungal packaging material.

Aureobasidiu (A.) mangrovei S. Nasr is a saprophytic black yeast-like fungus that was isolated from healthy leaves of Avicinnia marina in Iran [7]. The species has some resemblance to Aurebasidium pullulans which is also a yeast-like fungus capable of producing pullulan. Pullulan, an exopolysaccharide (EPS), is a linear homopolymer with a chemical structure composed of maltotriose subunits interconnected with $\alpha-1,6$ glucosidic linkages [8]. It is non-toxic, biodegradable, water soluble, non-carcinogenic, non-immunogenic and edible [9-12]. Pullulan has been applied in various fields, ranging from food manufacturing to pharmaceutical applications, and even used as a source of edible monosaccharaides. Pullulan is an excellent film former, producing a biodegradable film, which is heat sealable with good oxygen barrier properties [13]. As a result, pullulan film is sometimes referred to as "edible packaging." Coloring, flavoring, and other functional ingredients can be entrapped in the film matrix and are very stable. It has also been reported that pullulan can inhibit fungal growth in food [14]. Pullulan polymer is exploited commercially in the food industry, cosmetics, and manufacturing industries [15]. With its high viscosity, pullulan is also a candidate for enhanced oil recovery [16].

The objective of this research was to extract pullulan from Aureobasidium mangrovei, synthesize pullulanmediated AgNPs, characterize them and test their antibacterial activities against Staphylococcus aureus, E.coli, P. aeruginosa, and Bacillus cereus, and their antifungal activities against A. niger, A. flavus, A.ochraceus, Curvularia lunata, Penicillium spp., and Fusarium incarnatum.

\section{Materials and Methods}

The fungus was isolated from Nerium oleander leaf at Sultan Qaboos University botanic garden (Oman). It was identified using molecular techniques as A. mangrovei Nasr. It was deposited at CBS, the Netherlands, having an accession number CBS 142327 and a Sultan Qaboos University number, SQU 30. It was cultured in yeast malt extract broth, placed in a shaker at a speed of $200 \mathrm{rpm}$ and incubated at $25^{\circ} \mathrm{C}$ for 4 to 5 days [14]. This is the second report of the existence of this species.

To remove the fungal growth, the culture was centrifuged at $15000 \mathrm{~g}$ for $20 \mathrm{~min}$. For the extraction of pullulan, six $\mathrm{ml}$ of cold ethanol was transferred to three $\mathrm{ml}$ of cell-free culture medium in a test tube and kept at $4{ }^{\circ} \mathrm{C}$ for $12 \mathrm{~h}$. The precipitate was separated by centrifugation at $5000 \mathrm{rpm}$ at $4{ }^{\circ} \mathrm{C}$ for $10 \mathrm{~min}$. After removal of the residual ethanol, the precipitate was dissolved in distilled water and distributed in test tubes. The tubes were kept in the freezer at $-30{ }^{\circ} \mathrm{C}$ for 3 days and then lyophilized to get dry pullulan powder.

\subsection{Characterization of pullulan}

The extracted pullulan was scanned using Fourier Transform Infrared (FT-IR) spectroscopy [6]. It was compared with pullulan obtained from Sigma, USA (Number p4516). FT-IR spectra were recorded over a range of $4000-400 \mathrm{~cm}$ ${ }^{1}$. Ultraviolet-visible spectroscopy was used to identify the different concentrations of the pullulan.

\subsection{Synthesis of pullulan-mediated AgNPs}

For the synthesis of pullulan-mediated AgNPs, three different concentrations of pullulan (1,3 and 5\%) were mixed with $1 \mathrm{mM}$ freshly prepared silver nitrate solution using a magnetic stirrer. The formation of AgNPs was recognized when the color turned brown [17]. The solutions were stored at room temperature $\left(25^{\circ} \mathrm{C}\right)$.

Primary characterization of synthesized silver nanoparticles was carried out by UV-Vis spectroscopy. The stability of AgNPs was tested at different periods of time, $1.5 \mathrm{~h}, 3$ days, 7 days and 14 days. The UV-Vis absorbance spectrum of the solution was in the range of $300-700 \mathrm{~nm} ; \mathrm{AgNO}_{3}$ solution was used as a blank.

To examine the possible functional groups of pullulan which are responsible for the reduction and stabilization of AgNPs, FT-IR spectrum measurement was used. Dry AgNPs were prepared by lyophilization. Then, a sufficient amount of potassium bromide powder was added and placed in a mortar with a small amount of AgNPs of about the tip 
of the micro spatula. The sample was crushed until it was uniformly distributed throughout the KBr. The mixture was placed in a stainless steel container and a hydraulic press was applied to produce a disk. The disk was put in the sample holder which was located in the sample compartment for scanning. FT-IR spectra were recorded at $4000-400 \mathrm{~cm}^{-1}$.

Transmission electron microscopy (TEM) was used to determine the size, shape, morphology and distribution of AgNPs. For the analysis of TEM, a drop of AgNP-containing aqueous solution was placed on a carbon-coated copper grid, and then the drop was allowed to air dry completely prior to the TEM observations. At $300 \mathrm{kV}$ of accelerating voltage, TEM images of AgNPs were obtained using a transmission electron microscope [6].

\section{Results and Discussion}

\subsection{Production of pullulan}

A. mangrovei had grown well in yeast malt extract broth after five days of incubation. The fungus produced pullulan together with melanin. This is the first report for this species to produce pullulan. Many strains of $A$. pullulans reported in the literature produced melanin. Pigment accumulation typically occurs late in culture growth, possibly associated with the formation of chlamydospore [9]. For commercial production, companies prefer to use strains which are non-melanin producers (mutant strains) or other non-pigment producers. However, pullulan can be purified of melanin by the addition of activated charcoal or hydrogen peroxide, sodium permanganate or other chemicals, but this makes pullulan more expensive.

\subsection{Extraction of pullulan}

Pullulan is synthesized intracellularly and secreted into media by A. pullulans strains. Most pullulan producing A. pullulans strains were reported from tropical countries. Different strains have different abilities in pullulan production depending on their genetics and cultural conditions and pullulan production depends both on species and strain performance and the nature of the medium. The pullulan produced by different strains have ranged from $1.3 \mathrm{~g} / \mathrm{L}$ to 29 $\mathrm{g} / \mathrm{L}$ when harvested from a batch culture using stirred tank fermenter [18]. About $1.6 \mathrm{~g} / \mathrm{L}$ of pullulan was extracted from A. mangrovei after five days of incubation.

\subsection{Characterization of pullulan by FT-IR analysis}

The FT-IR analysis of pullulan produced by A. mangrovei was compared to pullulan obtained from Sigma. The strong absorption at $3435.75 \mathrm{~cm}^{-1}$ indicated that both the pullulans had some repeating units of -OH as in sugars (Fig. 1). The other strong absorption at $2920.23 \mathrm{~cm}^{-1}$ indicated a $-\mathrm{C}-\mathrm{H}$ bond, and the absorptions at $1633 \mathrm{~cm}^{-1} \mathrm{a}-\mathrm{COOH}$ bond, at $1383 \mathrm{~cm}^{-1}$ a C-OH bond, and at $1066 \mathrm{~cm}^{-1}$ the $-\mathrm{C}-\mathrm{O}$ bonds in the ketone compounds. The extracted pullulan peaks and the standard Sigma pullulan peaks were almost identical, which proves that our extracted compound from $A$. mangrovei was pullulan. Our results were similar to those of previous studies [19].

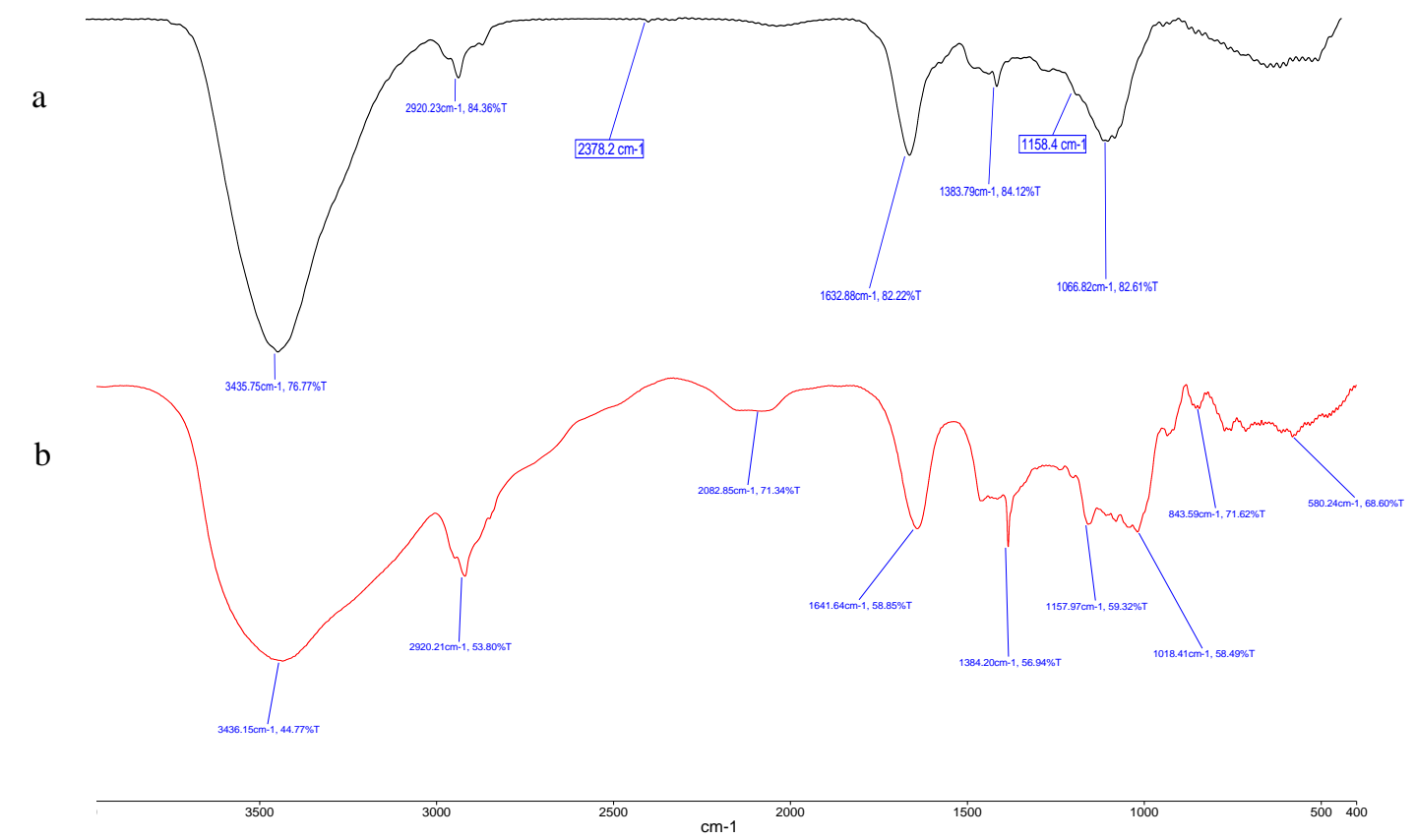

Figure 1. FT-IR of a) Standard Sigma pullulan and b) Extracted pullulan. 


\section{SYNTHESIS OF PULLULAN MEDIATED-SILVER NANOPARTICLES (AGNPS)}

\subsection{Characterization of AgNPs}

\subsubsection{UV -Vis spectroscopy}

By the addition of pullulan into silver nitrate, AgNPs were synthesized by the reduction of $\mathrm{Ag}^{+}$into $\mathrm{Ag}^{0}$. The colorless solution turned to brown, which indicated the formation of AgNPs [17, 20]. The synthesis of AgNPs were tested by UV-Vis spectroscopy. Pullulan concentration $(0.01 \%)$ formed AgNPs when mixed with $\mathrm{AgNO}_{3}$. A clear peak between 400-450 nm is an indication of AgNPs. The pullulan mediated AgNPs were tested for stability at $1.5 \mathrm{~h}, 3$ days, 7 days and 14 days. The results showed that they were stable at room temperature (Figure 2).

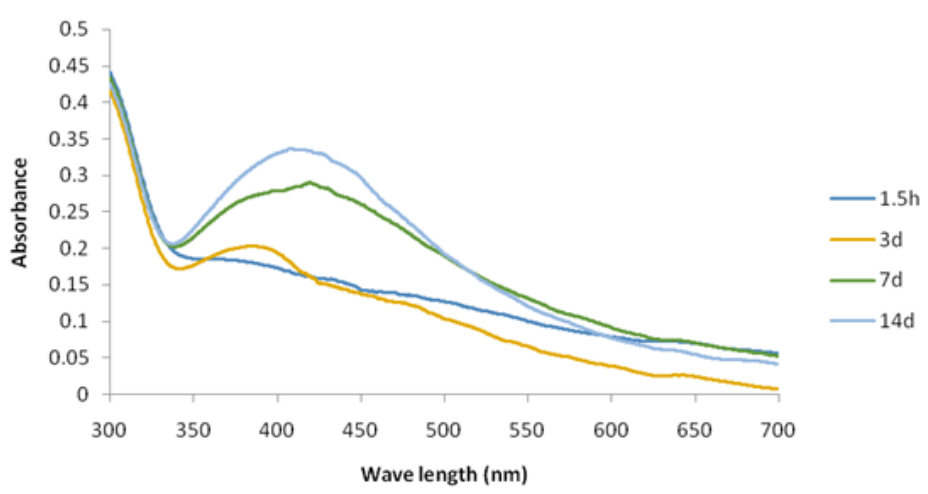

Figure 2. UV-Vis spectra of AgNPs at different periods.

\subsubsection{Transmission Electron Microscopy (TEM)}

TEM was used to visualize the size and shape of the pullulan mediated AgNPs. It is evident from Figure 3 that most of the synthesized particles were poly-dispersed, irregular in shape, and that most were spherical. The average diameter of the AgNPs was between 2-24 nm. The average size of the pullulan-mediated AgNPs was $9.76 \mathrm{~nm}$; this figure was obtained from measuring 45 particles. It was found that the pullulan produced spherical AgNPs had a size of between 50-55 nm. [17], while in another study, the average size of the spherical AgNPs was reported to be between 2$40 \mathrm{~nm}[6]$.
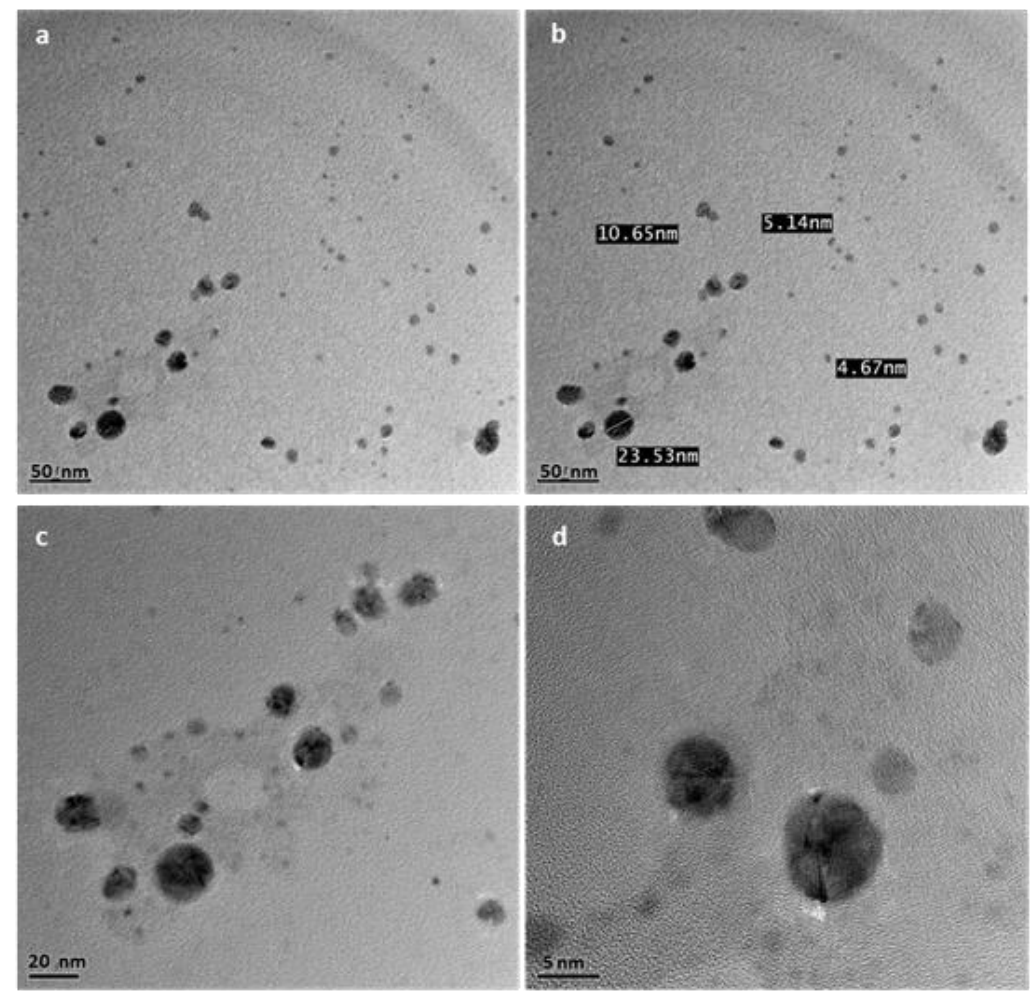

Figure 3. TEM micrograph showing the size of synthesized AgNPs. a,b) $50 \mathrm{~nm}$ scale, c) $20 \mathrm{~nm}$ scale and d) $5 \mathrm{~nm}$ scale. 


\subsubsection{Scanning Electron Microscope (SEM)}

The surface morphology of the AgNPs was investigated using a SEM. The micrographs in Figure 4 show that the synthesized AgNPs had rough surfaces, and were spherical with uneven, irregular beads of different sizes. The results indicate that the reduction process takes place at the surface. The rough surface may be an advantage for the immobilization of enzymes [6]. The micrograph shows that the nanoparticles did not aggregate, but were dispersed as individuals. This means that they were stable [6].
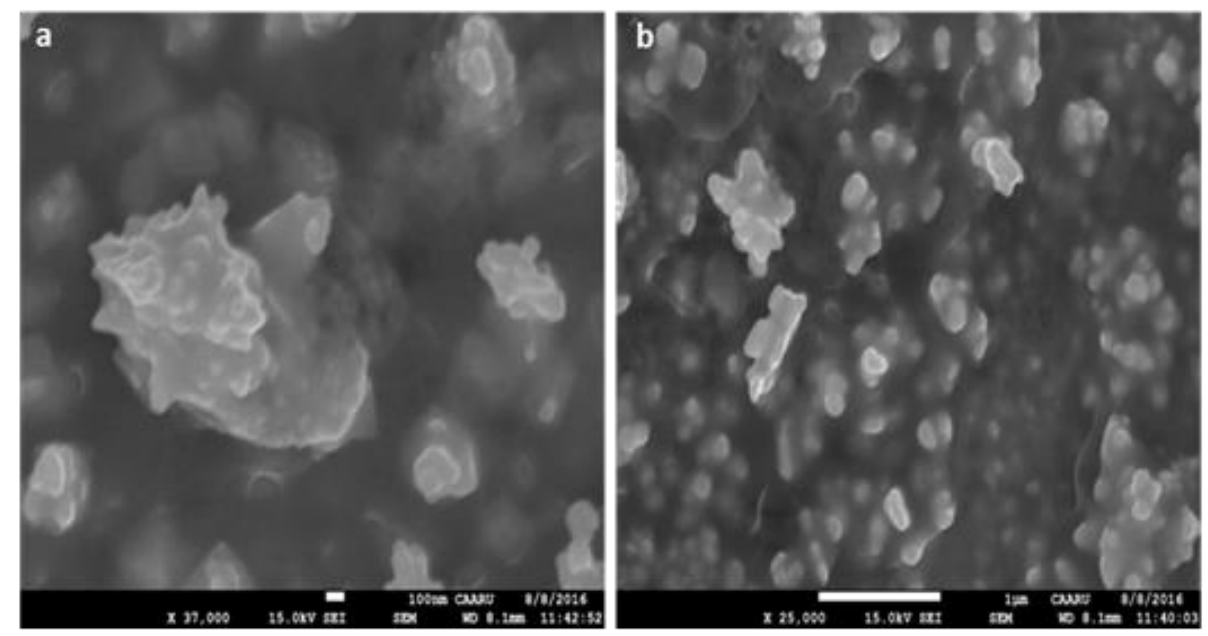

Figure 4. SEM imaging of AgNPs a) $100 \mathrm{~nm}$ scale; b) $1 \mu \mathrm{m}$ scale.

\subsubsection{Antibacterial activity of pullulan, $\mathrm{AgNO}_{3}$ and $\mathrm{AgNPs}$}

Pullulan has been reported to have some antimicrobial properties and has been used as a coating agent. In this study, pullulan, $\mathrm{AgNO}_{3}$ and $\mathrm{AgNPs}$ were tested against E. coli, S. aureus, B. cereus and P. aeruginosa obtained from The American Type Culture Collection (ATCC). Figure 5 shows that pullulan, $\mathrm{AgNO}_{3}$, and $\mathrm{AgNPs}$ have antimicrobial activity against E. coli, S. aureus, B. cereus and P. aeruginosa but that the degree of inhibition, as measured by the average of inhibition zones, differs. $\mathrm{AgNO}_{3}$ showed better antimicrobial activities against E.coli, B. cereus and P. aeruginosa compared to pullulan, and AgNPs, although not against $S$. aureus. The ANOVA test showed that there were no significant differences between $\mathrm{AgNO}_{3}$, pullulan and $\mathrm{AgNPs}$ against any of the bacteria tested. It has previously been reported that AgNPs can inhibit the growth of Gram negative bacteria such as $E$. coli and $P$. aeruginosa [6], while other researchers [17] have reported that Gram positive bacteria such as $S$. aureus can also be inhibited by the AgNPs. It has also been reported that [20] there was no antibacterial activity or inhibition when pullulan was tested on a film of $S$. aureus, L. monocytogenes, E. coli, and S. typhimurium, while other researchers [6] have found that only about $5 \%$ of $E$. coli and $P$. aeruginosa is inhibited by pullulan. Our pullulan, which was extracted for the first time from A. mangrovei, showed better antibacterial activity against both Gram negative and Gram positive bacteria.

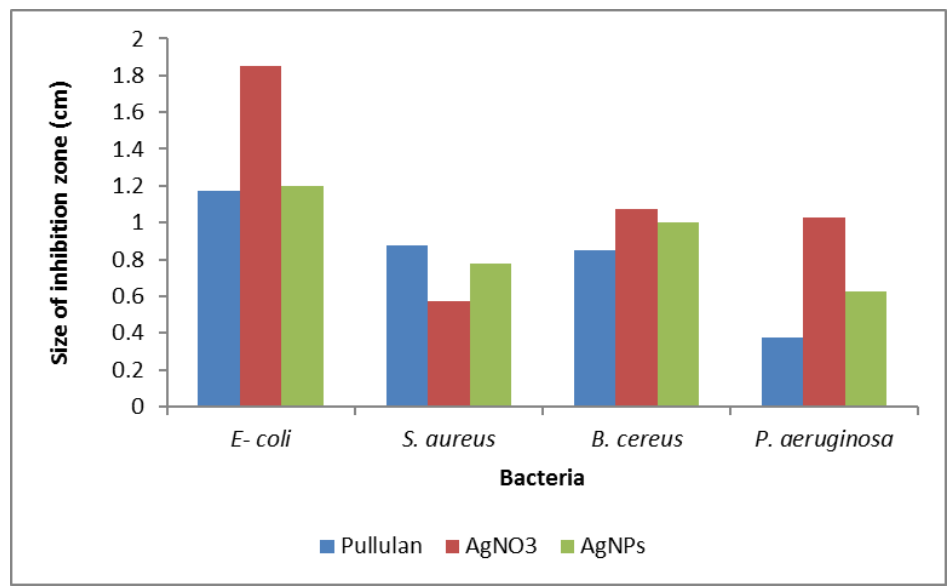

Figure 5. Antibacterial activity of pullulan, $\mathrm{AgNO}_{3}$ and $\mathrm{AgNPs}$ (Average diameter of the inhibition zones). 


\section{SYNTHESIS OF PULLULAN MEDIATED-SILVER NANOPARTICLES (AGNPS)}

\subsubsection{Antifungal activity of pullulan, $\mathrm{AgNO}_{3}$, and $\mathrm{AgNPs}$}

The antifungal activity of pullulan, $\mathrm{AgNO}_{3}$, and $\mathrm{AgNPs}$ was tested against $C$. lunata, F. incarnatum, A. niger, A. flavus, A. ochraceus and Penicillium sp. obtained from Sultan Qaboos University Biology Department Culture Collection. Figure 6 shows that pullulan, $\mathrm{AgNO}_{3}$, and $\mathrm{AgNPs}$ have antifungal activities against $C$. lunata, $F$. incarnatum, A. niger, A. flavus, A. ochraceus and Penicillium. ANOVA testing showed that there were significant differences between the anti-fungal effects of $\mathrm{AgNO}_{3}$, pullulan and $\mathrm{AgNPs}$, where $\mathrm{AgNO}_{3}$ had a higher inhibitory effect compared to both the AgNPs and pullulan, and that AgNPs were more strongly inhibitory than pullulan.Our results are similar to those of previous researchers [6] who showed that AgNPs inhibited Penicillium sp. and Aspergillus sp. Others [21] have also found that AgNPs inhibit some fungal pathogens such as A. niger, A. flavus, Penicillium sp. and Curvularia sp., but their reducing agent was the leaf extract of Cassia roxburghii and not pullulan.

The pullulan-mediated AgNPs could be used as an antifungal agent in controlling various mycotoxigenic fungi such as A. flavus, which produces aflatoxin that causes liver cancer, A. ochraceus, which produces ochratoxin that causes kidney damage, and plant pathogens such as $F$. incarnatum and food spoilage fungi such as Penicillium and Rhizopus.

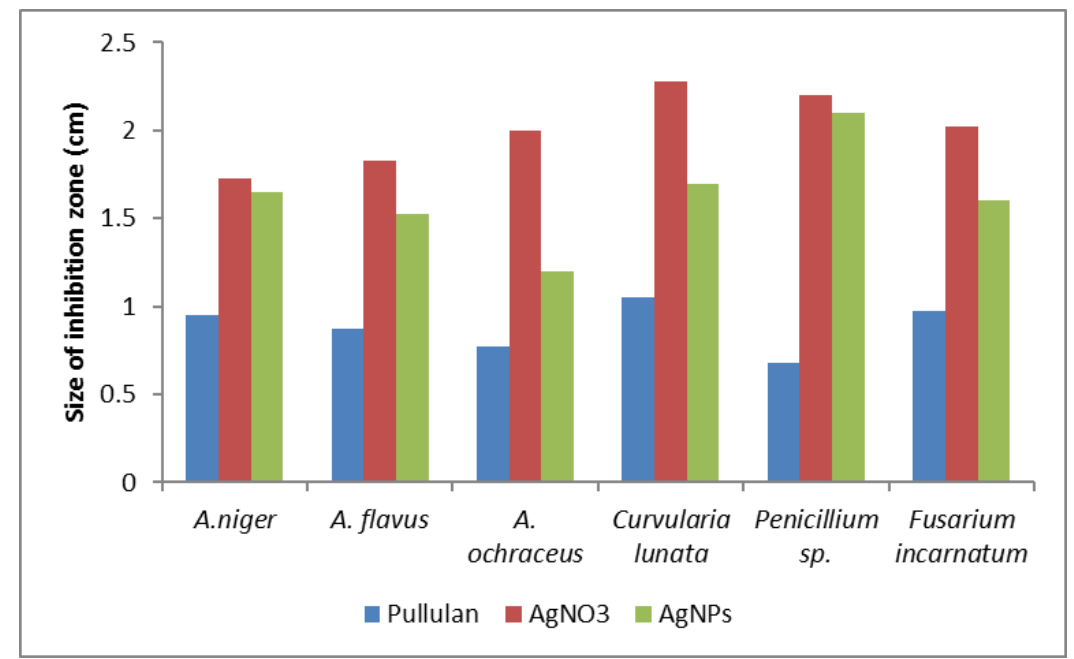

Figure 6. Antifungal activity of pullulan, $\mathrm{AgNO}_{3}$, and $\mathrm{AgNPs}$.

\section{Conclusion}

Pullulan-mediated AgNPs are of particular interest because of their ease of production, high antimicrobial activity, and ability to be incorporated into a diverse range of products. With the increasing number of antibioticresistant strains of bacteria and silver's high toxicity to humans, the use of AgNPs as an antimicrobial agent is an exciting topic with a great deal of relevance to many fields of study and to industry. Since only two papers have been published in using pullulan as reducing agent for the synthesis of nanoparticles, this study makes a contribution to this field. Pullulan was extracted for the first time from A. mangrovei, which was isolated from the SQU botanic garden. This species has shown better antibacterial and antifungal activities than other published strains of A. pullulans.

\section{Conflict of interest}

The authors declare no conflict of interest.

\section{Acknowledgement}

The authors would like to acknowledge the Central Analytical and Applied Research Unit (CAARU), Department of Chemistry at the College of Science and the College of Medicine, Sultan Qaboos University, for giving access to their facilities.

\section{References}

1. Rauwel, P., Küünal, S., Ferdov, S. and Rauwel, E. A review on the green synthesis of silver nanoparticles and their morphologies studied via TEM. Advances in Materials Science and Engineering, 2015, 2015, 682749. 
2. Pinto, R.J.B., Almedia, A., Fernandes, S.C., Freire, C.S., Silvestre, A.J., Neto, C.P. and Trindade, T. Antifungal activity of transparent nanocomposie thin films of pullulan and silver against Aspergillus niger. Colloids and Surfaces B Biointerfaces, 2013, 103,143-148.

3. Awwad, Akl. M., Salem, N.M. and Abdeen, A.O. Green synthesis of silver nanoparticles using carbo leaf extract and its antimicrobial activity. International Journal of Industrial Chemistry, 2013, 4, 29.

4. Krishnaraj, C., Jagan, E.G., Rajasekar, S., Selvakumar, P., Kalaichelvan, P.T. and Mohan, N. Synthesis of silver nanoparticles using Acalypha indica leaf extracts and its antibacterial activity against water borne pathogens. Colloids and Surfaces B: Biointerfaces, 2010, 76, 50-55.

5. Naqvi, S.Z.H, Kiran, U. and Ali, M.I. Combined efficacy of biologically synthesized silver nanoparticles and different antibiotics against multidrug-resistant bacteria. International journal of Nanomedicine, 2013, 8, 31873195.

6. Kanmani, P., and Lim, S.T. Synthesis and characterization of pullulan-mediated silver nanoparticles and its antimicrobial activities. Carbohydrate Polymers, 2013, 97, 421-428.

7. Nasr, S., Mohammadimehr, M., Vaghei, M.G., Amoozegar, M.A. and Fazeli, S.A.S. Aureobasidium mangrovei sp. nov., an ascomycetes species recovered from Hara protected forests in the Persian Gulf, Iran. Antonie van Leeuwenhoek, 2018, 111, 1697-1705.

8. Bender, H. and Wallenfels, K Untersuchungen an Pullulan. II. Spezifischer Abbau durch ein bakterielles Enzym. Biochem Z, 1961, 334, 79-95.

9. Leathers, T.D. Pullulan. Biopolymers Online. 2005. http://onlinelibrary.wiley.com/doi/10.1002/3527600035.bpol6001/full

10. Choudhury, A.R., Saluja, P. and Prasad, G.S. Pullulan production by an osmotolerant Aureobasidium pullulans RBF-4A3 isolated from flowers of Casesuliaaxillaris. Carbohydrate Polymers, 2010, 83, 1547-1552.

11. Pollock, T.J., Thorne, L. and Armentrout, R.W. Isolation of new Aureobasidium strains that produce highmolecular -weight pullulan with reduced pigmentation. Applied and Environmental Microbiology, 1991, 58, 377883.

12. Kang, J.X., Chen, X.J., Chen, W.R., Li, M.S., Fang, Y., Li, D.S., Ren, Y.Z. and Liu, D.Q. Enhanced production of pullulan in Aureobasidium pullulans by new process of genome shuffling. Process Biochemistry, 2010, 46, 792795.

13. Yuen, S. Pullulan and its applications. Process Biochemistry, 1974, 9-7, 9.

14. Cheng, K.C., Demirci, A. and Catchmark, J.M. Pullulan: biosynthesis, production, and applications. Applied Microbiology and Biotechnology, 2011, 92, 29-44.

15. Prasongsuk, S. Pullulan production by tropical isolates of Aureobasidium pullulans. Journal of Industrial Microbiology and Biotechnology, 2006, 4(1), 55-61.

16. Iyer, A., Mody, K. and Jha, B. Biosorption of heavy metals by a marine bacterium. Marine Pollution Bulletin, 2005, 50, 340-343.

17. Coseri, S., Spatareanu, A., Sacarescu, L., Rimbu, C., Suteu, D., Spirk, S. and Harabagiu, V. Green synthesis of the silver nanoparticles mediated by pullulan and 6-carboxypullulan. Carbohydrate Polymers, 2015, 116, 9-17.

18. Ravella, S.R., Quiñones, T.S., Retter, A., Heiermann, M., Amon, T. and Hobbs, P.J. Extracellular polysaccharide (EPS) production by a novel strain of yeast-like fungus Aureobasidium pullulans. Carbohydrate Polymers, 2010, 82, 728-732.

19. Mishra, B. and Vuppu S. A Study on downstream processing for the production of pullulan by Aureobasidium pullulans-SB-01 from the fermentation broth. Research Journal of Recent Sciences, 2013, 2, 16-19.

20. Morsy, M.K., Khalaf, H.H., Sharoba, A.M., El-Tanahi H.H. and Cutter, C.N. Incorporation of essential oils and nanoparticles in pullulan films to control foodborne pathogens on meat and poultry products. Journal of Food Science, 2014, 79, 675-84.

21. Balashanmugam, P., Balakumaran, M.D., Murugan, R., Dhanapal, K. and Kalaichelvan, P.T. Phytogenic synthesis of silver nanoparticles, optimization and evaluation of in vitro antifungal activity against human and plant pathogens. Microbiological Research, 2016, 192, 52-64.

Received 11 February 2019

Accepted 9 June 2019 